\title{
Does Cosmic-Ray-Induced Heterogeneous Chemistry Influence Stratospheric Polar Ozone Loss?
}

\author{
Rolf Müller* and Jens-Uwe Grooß \\ ICG-1, Forschungszentrum Jülich, 52425 Jülich, Germany
}

(Received 20 May 2009; published 24 November 2009)

\begin{abstract}
Cosmic-ray (CR) -induced heterogeneous reactions of halogenated species have been suggested to play the dominant role in causing the Antarctic ozone hole. However, measurements of total ozone in Antarctica do not show a compact and significant correlation with CR activity. Further, a substantial CR-induced heterogeneous loss of chlorofluorocarbons is incompatible with multiyear satellite observations of $\mathrm{N}_{2} \mathrm{O}$ and $\mathrm{CFC}-12$. Thus, CR-induced heterogeneous reactions cannot be considered as an alternative mechanism causing the Antarctic ozone hole.
\end{abstract}

DOI: 10.1103/PhysRevLett.103.228501

PACS numbers: 92.70.Cp, 82.33.Tb, 82.65.+r

The discovery of the Antarctic ozone hole in 1985 [1] came as a great surprise (e.g., [2,3]) because, at that time, none of the major physicochemical mechanisms responsible for its occurrence, were known. Today, it is well established $[2,4]$ that the precipitous loss of $\mathrm{O}_{3}$ in Antarctic spring is caused by a chain of events starting in autumn with cooling of the polar stratosphere and the formation of a strong wind jet that, to a great extent, isolates the polar air-mass from midlatitudes. This isolated, rotating air mass is referred to as the polar vortex. In winter, when stratospheric temperatures are low enough, polar stratospheric clouds (PSC) form providing surfaces which allow heterogeneous reactions to occur. These reactions convert chlorine from so-called reservoir species (mostly $\mathrm{HCl}$ and $\mathrm{ClONO}_{2}$ ), to an active, $\mathrm{O}_{3}$-destroying form $[2,5]$. The $\mathrm{O}_{3}$ destruction occurs in catalytic cycles, in the Antarctic stratosphere mainly through a cycle involving chlorine peroxide $(\mathrm{ClOOCl})[2,6]$. The $\mathrm{O}_{3}$ destruction stops either when extremely low mixing ratios of $\mathrm{O}_{3}$ are reached $[7,8]$ or when temperatures rise above the PSC existence threshold, so that the chlorine reservoir species are reformed at the cost of active chlorine, a process which causes $\mathrm{O}_{3}$ depletion to halt. Uncertainties still exist; for example, the exact mechanism of heterogeneous chlorine activation is being debated (e.g., [9]) and chemical rate constants that strongly impact the speed at which $\mathrm{O}_{3}$ loss proceeds in the polar stratosphere are not yet satisfactorily known $[6,10,11]$.

Recently, Lu [12] put forward the idea that cosmic rays (CR) lead to the presence of trapped electrons in PSC particles followed by dissociative electron attachment to both chlorine reservoir species $\left(\mathrm{HCl}\right.$ and $\left.\mathrm{ClONO}_{2}\right)$ and chlorofluorocarbons (CFC). This issue has been debated before [13-18]. Lu [12] reports "reliable satellite data in the period of 1980-2007 covering two full 11-yr cosmic-ray (CR) cycles, clearly showing the correlation between CRs and ozone depletion, especially the polar ozone loss (hole) over Antarctica." Here we revisit the issue showing that, first, in the Antarctic there is no strong and significant correlation between CR activity and polar $\mathrm{O}_{3}$ loss, second, that the 11-year modulation in tropical and midlatitude $\mathrm{O}_{3}$ is both well known and unrelated to polar processes, and, third, that CR-driven reactions can only be a possible addition to the set of processes known to cause the $\mathrm{O}_{3}$ hole and not an alternative mechanism.

$\mathrm{Lu}$ [12] presents correlations between CR intensity and different spatial and temporal averages of total column $\mathrm{O}_{3}$ measurements, namely, annual mean total $\mathrm{O}_{3}$ in the latitude band $0^{\circ}-60^{\circ} \mathrm{S}$, October mean total column $\mathrm{O}_{3}$ poleward of $60^{\circ} \mathrm{S}$, and annual mean total $\mathrm{O}_{3}$ at two Antarctic stations (Halley and Vernadsky). He states that the "....appearance of the polar $\mathrm{O}_{3}$ hole is known to have a consequent effect on annual total $\mathrm{O}_{3}$ in the polar area and globally." While this statement is somewhat misleading (the Antarctic $\mathrm{O}_{3}$ hole is not known to have an effect on the tropics [4]), it is important to note that the proposed CR effect is a polar winter and spring effect as it is coupled to the occurrence of PSC. Therefore, its signal (and the correlation with $\mathrm{CR}$ intensity) should be strongest in the Antarctic in spring. In the annual average of polar total $\mathrm{O}_{3}$ [[12], Fig. 3], the signal of the PSC-driven CR effect will be damped by other unrelated processes, for example, the strong nitrogen-oxide-driven chemical polar $\mathrm{O}_{3}$ loss in summer [19]. Further, even in austral spring, the Vernadsky station is located at the edge of the $\mathrm{O}_{3}$ hole region so that these measurements are influenced by both polar and midlatitude processes.

It is well known $[4,20]$ that the solar cycle has an impact on total $\mathrm{O}_{3}$ outside the polar regions, in the tropics and in midlatitudes [[12], Fig. 1]. The solar-cycle variation of tropical $\mathrm{O}_{3}$ is also detected in vertical profile measurements of upper stratospheric $\mathrm{O}_{3}$ [21]. Further, the solarcycle variation of tropical and midlatitude total $\mathrm{O}_{3}$ was observed in ground-based data sets covering the years 1960-1980, when no substantial Antarctic $\mathrm{O}_{3}$ loss was observed [22].

For recent decades, the detection of the solar-cycle signal has been complicated by the fact that the eruptions of El Chichón (1982) and Mt. Pinatubo (1991) occurred near the declining phases of the solar cycle in 1982-1984 and 1992-1994, so that there is the possibility of aliasing 


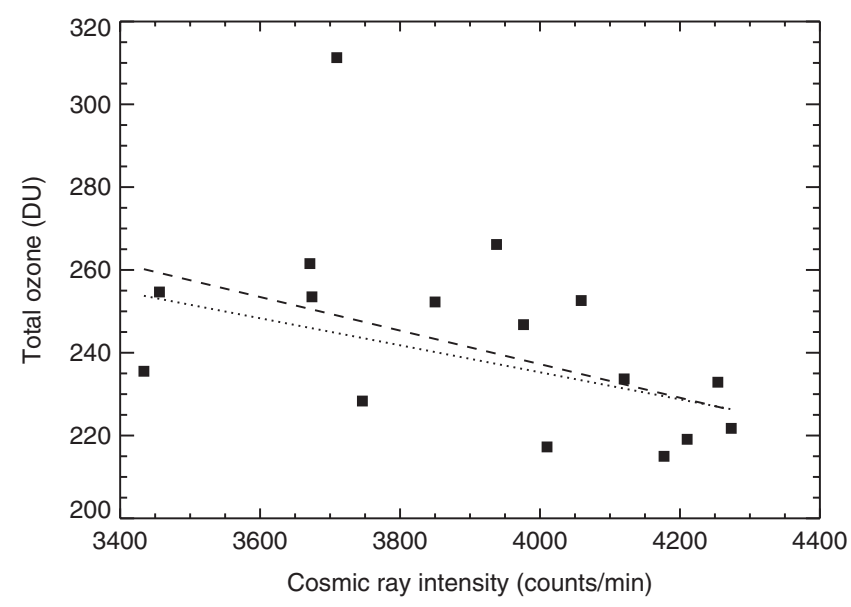

FIG. 1. Relation between cosmic-ray intensity (from Ref. [17]) and mean total column $\mathrm{O}_{3}$ for October poleward of $60^{\circ} \mathrm{S}$ for 1990-2005 (from Refs. [27,28]). Dashed line shows the linear fit to all data points, dotted line shows the linear fit to the data points excluding 2002 (column $\mathrm{O}_{3} 311$ DU). We assume an upper limit of the uncertainty of the mean total $\mathrm{O}_{3}$ of $5 \%$.

volcanic and solar-cycle related effects on column $\mathrm{O}_{3}$ [23]. Moreover, a periodic fluctuation of global total $\mathrm{O}_{3}$ is caused by the quasi-biennial oscillation in tropical stratospheric winds $[4,24]$, which has to be taken into account when analyzing the 11-year solar-cycle signal in column $\mathrm{O}_{3}$ [25]. Thus, the 11-year variation of total column $\mathrm{O}_{3}$ in the extra-polar region of the southern hemisphere $\left(0^{\circ}-\right.$ $60^{\circ} \mathrm{S}$ ) is highly unlikely to be related to polar processes.

Therefore, in the following, we focus on the comparison of the percentage variation of $\mathrm{CR}$ intensity and October mean total $\mathrm{O}_{3}$ poleward of $60^{\circ} \mathrm{S}$ during the period 19902007 [[12], Fig. 2]. In the discussion of this figure, it is tacitly assumed that the October mean total $\mathrm{O}_{3}$ poleward of $60^{\circ} \mathrm{S}$ constitutes a suitable measure of chemical $\mathrm{O}_{3}$ loss. While this quantity is certainly strongly influenced by chemical loss in the $\mathrm{O}_{3}$ hole, there are also other factors unrelated to chemistry that influence this quantity, in particular, the size and the shape of the polar vortex. Sophisticated measures of chemical polar $\mathrm{O}_{3}$ loss have been developed [26] including additional information to disentangle the impact of transport and chemistry on $\mathrm{O}_{3}$, which should be employed whenever possible. But even when only total $\mathrm{O}_{3}$ measurements are available, a better measure of chemical $\mathrm{O}_{3}$ loss than October mean total $\mathrm{O}_{3}$ poleward of $60^{\circ} \mathrm{S}$ is available [27], namely, the minimum of daily average total $\mathrm{O}_{3}$ poleward of $63^{\circ}$ equivalent latitude. A time series of this quantity over the years 1979-2005 shows no correlation with solar intensity or CR activity [17]. The data set used in Ref. [17] has been criticized as "noisy", considering the entire temporal variability in the time series of about $20 \%$ as "noisy level" [18]. However, the data set used in Ref. [17] and in the present work is essentially the same as the TOMS-OMI data set analyzed by Lu [12], the TOMS-OMI data are solely corrected for consistency between the different se- ries of instruments and, when TOMS-OMI data are not available, data gaps are filled using other instruments [27,28]. This allows the years 1993 and 1995 to be included in the analysis which are omitted in Ref. [12]. The corrections applied to the total $\mathrm{O}_{3}$ data set are small [28] and are not relevant for the large interannual variability which is of interest here.

In Fig. 1 we show the relation between CR-intensity and the mean total column $\mathrm{O}_{3}$ for October poleward of $60^{\circ} \mathrm{S}$ for the time period 1990-2005. There is only a moderate variation of polar $\mathrm{O}_{3}$ with $\mathrm{CR}$ intensity over this time period (Fig. 1, dashed line) which only extends over less than two solar cycles. The variation is even weaker when the year 2002 is excluded from the fit (Fig. 1, dotted line), a year which shows an extremely unusual dynamics of the polar vortex $[4,29]$. The (linear Pearson) correlation coefficient between $\mathrm{CR}$ intensity and the mean total column Antarctic $\mathrm{O}_{3}$ for October (for the data in Fig. 1) for the entire time series (1979-2005) is $r=-0.37$, for the time period 1990-2005, $r=-0.49$, and for the time period 1990-2005 excluding the year 2002, $r=-0.52$. The reduced $\chi^{2}$ goodness of fit parameter for the fit for 19902005 is 2.7 and, if 2002 is excluded it is 1.6. The probability of such a poor $\chi^{2}$ value occurring by chance is $6.4 \times 10^{-4}$ and $8.7 \times 10^{-2}$, respectively. We assumed an upper limit of the uncertainty of the monthly mean total column Antarctic $\mathrm{O}_{3}$ of $5 \%$. The correlation coefficient for the data shown in Fig. 2 of Ref. [12] is $r=-0.37$. Thus, there is no solid statistical foundation for a linear correlation between CR intensity and the mean total column Antarctic $\mathrm{O}_{3}$ in October for the years since 1990 .

Moreover, the enhancement of the stratospheric aerosol loading caused by the eruption of Mt. Pinatubo in $1991 \mathrm{had}$ a strong impact on polar stratospheric $\mathrm{O}_{3}[7,30,31]$; in particular, the low Antarctic $\mathrm{O}_{3}$ values in the 1990s (during an ascending phase of CR intensity) have been associated with the Mt. Pinatubo eruption [7,30]. Thus, the coincidence of a strongly enhanced stratospheric aerosol layer and increasing CR intensity in the 1990s could lead to a spurious correlation via an aliasing effect. Furthermore, Lu [12] assumes that "from 1992 up to now [...] the total halogen amount of the stratosphere [...] is nearly constant ...". This is not correct; the Antarctic stratospheric halogen burden for 1990 is $\sim 20 \%$ below the peak value in $\sim 2001$ and in 2020 is predicted to be $\sim 10 \%$ below the peak value $[32,33]$.

$\mathrm{Lu}$ [12] also puts forward a linear relation between CR intensity and total $\mathrm{O}_{3}$ variation as a method of predicting the future development of the $\mathrm{O}_{3}$ hole. This relation is deduced by first reducing the variability of the October polar data (1990-2007) by a factor of 5, the annual polar data (1990-2007) by a factor of 2.5, then combining the two data sets with the (1980-2007) $0-60^{\circ}$ total $\mathrm{O}_{3}$ data into one single data set. A linear fit to the combined data set (Ref. [12], red line in Fig. 4) is then scaled up again by a factor of 5 to obtain a prediction of October polar $\mathrm{O}_{3}$ levels. This procedure is questionable insofar as three very differ- 


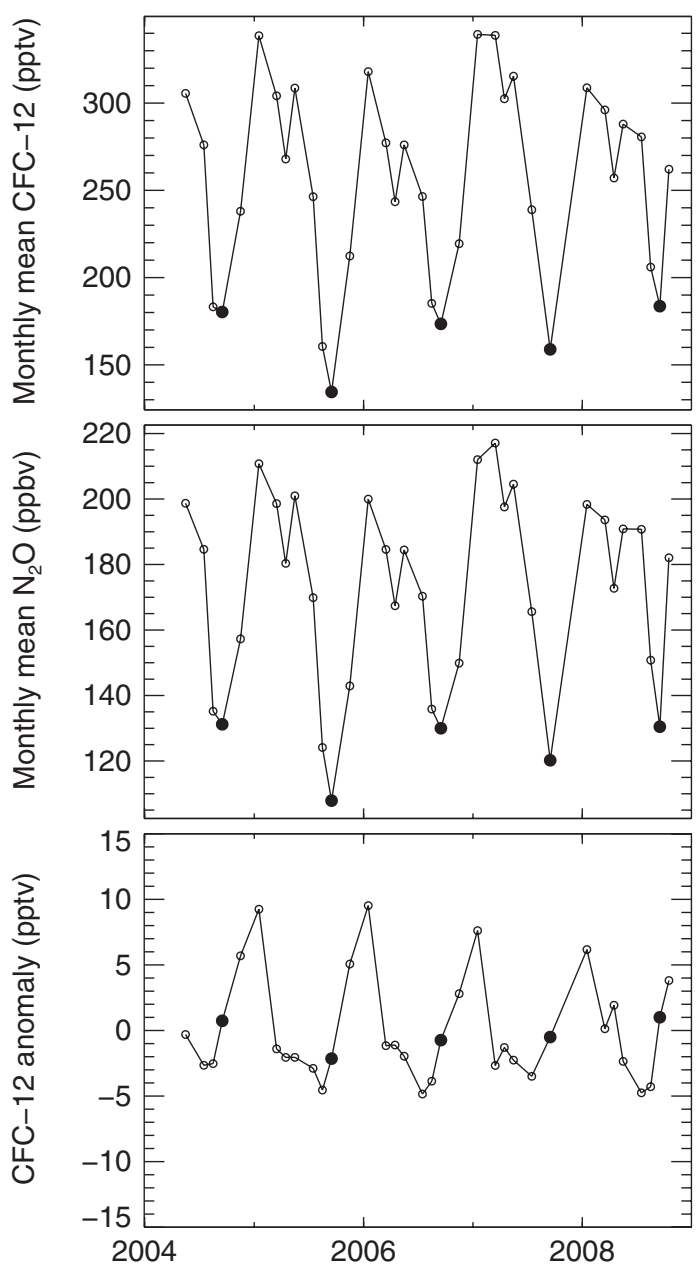

FIG. 2. Time series of ACE-FTS satellite measurements of CFC-12 (top) and $\mathrm{N}_{2} \mathrm{O}$ (middle) as well as the CFC-12 anomaly relative to $\mathrm{N}_{2} \mathrm{O}$ (bottom) at $18.5 \mathrm{~km}$, monthly averaged poleward of $60^{\circ} \mathrm{S}$. September data are marked by filled circles. The CFC-12 anomaly is deduced by first calculating from ACEFTS data poleward of $60^{\circ} \mathrm{S}$ between 10 and $25 \mathrm{~km}$ an empirical relation: $\quad \mu_{\mathrm{CFC}-12}=4.77+0.558 \mu_{\mathrm{N}_{2} \mathrm{O}}+0.00741\left(\mu_{\mathrm{N}_{2} \mathrm{O}}\right)^{2}-$ $1.31 \times 10^{-5} \times\left(\mu_{\mathrm{N}_{2} \mathrm{O}}\right)^{3}$, where $\mu_{\mathrm{N}_{2} \mathrm{O}}$ and $\mu_{\mathrm{CFC}-12}$ are volume mixing ratios in parts per billion (ppbv) and parts per trillion (pptv), respectively. Then, the CFC-12 anomaly is calculated for each data point as the difference of the observed CFC-12 and CFC-12 derived from the correlation using the observed $\mathrm{N}_{2} \mathrm{O}$. The latitude of the ACE-FTS observations in September is $74^{\circ} \pm 6^{\circ} \mathrm{S}$ due to the satellite's viewing geometry.

ent data sets are combined into one single fit, so that the properties of one data set influence the prediction of the other. In particular, the known 11-year solar cycle signal of extrapolar $\mathrm{O}_{3}$ may influence the prediction of October Antarctic $\mathrm{O}_{3}$. Also, the TOMS-OMI total column $\mathrm{O}_{3}$ averages reported by $\mathrm{Lu}[12]$ are incorrect. The reported average $\mathrm{O}_{3}$ column for October 1992 between $60^{\circ} \mathrm{S}$ and $90^{\circ} \mathrm{S}$ is 219 Dobson units (DU), while the correct value for this quantity obtained from Nimbus-7 TOMS Version 8 data is 254 DU, when first averaged over time and then, area weighted, over area.
An important assertion made by $\mathrm{Lu}$ [12] is that his results "cannot be explained by the photochemical model" and that one is forced "to conclude that the CR-driven electron-induced reaction is the dominant mechanism for causing the polar $\mathrm{O}_{3}$ hole." This gives the impression that two independent and competing mechanisms exist that may explain polar stratospheric $\mathrm{O}_{3}$ depletion. However, the effect of the proposed CR-driven heterogeneous chemistry can only be a destruction of CFC or chlorine reservoir species on PSC surfaces and, therefore, only an enhanced activation of chlorine. Ozone destruction, also in the CR mechanism, can only occur through the action of the activated chlorine on $\mathrm{O}_{3}$ in catalytic loss cycles. Therefore, clearly, the $\mathrm{CR}$ mechanism proposed by $\mathrm{Lu}$ [12] cannot resolve the discrepancy between the simulated and observed $\mathrm{O}_{3}$ loss that was raised by recent measurements of the $\mathrm{ClOOCl}$ cross sections $[6,10]$. This issue is more likely to be resolved by new laboratory work [34].

A fundamental problem with the $\mathrm{CR}$ mechanism is that a direct, linear relation between $\mathrm{CR}$ activity and polar $\mathrm{O}_{3}$ loss is assumed [[12], Fig. 4]. However, for "the regulating effect of CRs on $\mathrm{O}_{3}$ loss" [12] to become manifest, it needed to be demonstrated, first, that there is a regulating effect of CRs on low-energy electrons in PSC, and, second, that the density of low-energy electrons in PSC is rate limiting for the heterogeneous CR-induced destruction of $\mathrm{CFC}, \mathrm{HCl}$, or $\mathrm{ClONO}_{2}$. There is some support from laboratory experiments for the latter step, but none of these experiments have been conducted under stratospheric conditions [35].

If one nonetheless assumes that heterogeneous chlorine activation is regulated by CR activity, this in no way implies that also polar $\mathrm{O}_{3}$ loss depends linearly on chlorine activation. For example, in the 1990s, Antarctic $\mathrm{O}_{3}$ loss was frequently saturated in the sense that $\mathrm{O}_{3}$ was essentially zero in extended regions of the $\mathrm{O}_{3}$ hole $[4,7,8]$; under such conditions, no chemical effect, whether a greater stratospheric chlorine burden, a more rapid chlorine activation, or more efficient catalytic $\mathrm{O}_{3}$ loss cycles, can lead to enhanced $\mathrm{O}_{3}$ destruction.

If $\mathrm{CFC}$ were destroyed by CR-induced heterogeneous chemistry, the effect should be visible in measurements of CFC. Figure 2 shows a time series of CFC-12 (top) and $\mathrm{N}_{2} \mathrm{O}$ mixing ratios (middle) at $18.5 \mathrm{~km}$ from more than four years of measurements from the Atmospheric Chemistry Experiment (ACE-FTS) on SCISAT-1 [36]. All data poleward of $60^{\circ} \mathrm{S}$ are monthly averages. An annual cycle is clearly visible with the lowest observations in September (filled circles) with very similar behavior for $\mathrm{N}_{2} \mathrm{O}$ and CFC-12. The September air masses spent the longest time in the stratosphere, so that about two thirds of the CFC-12 originating from the troposphere has been photolytically dissociated. Because of interannual variability, both of atmospheric dynamics and latitude coverage of the satellite observations, the September $\mathrm{CFC}-12$ values vary as do the $\mathrm{N}_{2} \mathrm{O}$ values. Therefore, we deduced the CFC-12 anomaly relative to $\mathrm{N}_{2} \mathrm{O}$ (bottom panel of 
Fig. 2). If the proposed CR-mechanism caused significant additional dissociation of CFC-12 in the polar stratosphere, a decrease of the CFC-12 anomaly with time should be seen for the September values (when PSCs occur) with increasing CR intensity. However, between 2004 and 2008, when the CR intensity increased significantly, no corresponding decrease in the CFC-12 anomaly is observed.

Further, Lu [12] states that "...the solar-cycle effect [that is the "photochemical model"] should also predict that the higher solar intensity would lead to more destruction of CFCs and thus produce more active $\mathrm{Cl}$ to destroy $\mathrm{O}_{3}$ " [12]. This argument is flawed because the air in the polar regions has spent about five to six years in the stratosphere [32] so that the photochemical degradation of CFC is not controlled by the solar activity in a particular year. Moreover, the fact that the $\mathrm{CR}$-induced ionization rate peaks in the same region where temperatures are low enough for PSC to exist $[12,13,18]$ does not provide any support for the CR mechanism [15,33]. Finally, Lu [12] misinterprets model projections of the future development of polar $\mathrm{O}_{3}$ [4]. The models neither predict a monotonic recovery of polar total $\mathrm{O}_{3}$ since 2000 , nor are the predictions of total $\mathrm{O}_{3}$ over $60^{\circ} \mathrm{S}$ to $60^{\circ} \mathrm{N}$ and Antarctic springtime $\mathrm{O}_{3}$ inconsistent with observed data (see also Ref. [33]).

In summary, we have demonstrated here that the 11-year modulation in tropical and midlatitude $\mathrm{O}_{3}$ is both well known and unrelated to polar processes. It is rather driven by the 11-year cycle in solar radiation. Further, we have shown that in the Antarctic a compact and significant correlation does not exist between CR activity and chemical $\mathrm{O}_{3}$ loss in spring (i.e., the $\mathrm{O}_{3}$ hole). Moreover, a significant CR-induced heterogeneous loss of CFCs is incompatible with recent, multiyear satellite observations of CFC-12. However, CR activity may possibly impact the heterogeneous activation of the chlorine reservoir species $\mathrm{HCl}$ and $\mathrm{ClONO}_{2}$ [16,37], a possibility that should be further explored in laboratory studies under stratospheric conditions. In any case, CR-driven heterogeneous reactions can only be considered as a possible addition to the set of processes known to cause the Antarctic $\mathrm{O}_{3}$ hole and not as an alternative mechanism.

We thank F. Rohrer for comments and for calculating the correlation coefficient of the data in Fig. 2 of Ref. [12]. ACE-FTS is funded by the Canadian Space Agency.

*ro.mueller@fz-juelich.de

[1] J. C. Farman, B. G. Gardiner, and J. D. Shanklin, Nature (London) 315, 207 (1985).

[2] S. Solomon, Rev. Geophys. 37, 275 (1999).

[3] R. Müller, Meteorol. Z. 18, 3 (2009).

[4] WMO, Scientific Assessment of Ozone Depletion: 2006 Global Ozone Research and Monitoring Project Report No. 50, Geneva, Switzerland, 2007.
[5] T. Peter, Annu. Rev. Phys. Chem. 48, 785 (1997).

[6] M. von Hobe et al., Atmos. Chem. Phys. 7, 3055 (2007).

[7] S. Solomon et al., J. Geophys. Res. 110, D21311 (2005).

[8] S. Solomon, R. W. Portmann, and D. W. J. Thompson, Proc. Natl. Acad. Sci. U.S.A. 104, 445 (2007).

[9] K. S. Carslaw, T. Peter, and R. Müller, Geophys. Res. Lett. 24, 1747 (1997); B. F. Henson et al., J. Chem. Phys. 121, 8486 (2004).

[10] F. D. Pope et al., J. Phys. Chem. A 111, 4322 (2007).

[11] M. von Hobe, Science 318, 1878 (2007).

[12] Q.-B. Lu, Phys. Rev. Lett. 102, 118501 (2009).

[13] Q.-B. Lu and L. Sanche, Phys. Rev. Lett. 87, 078501 (2001).

[14] Q.-B. Lu and L. Sanche, Phys. Rev. Lett. 89, 219802 (2002); 89, 219804 (2002); N. R. P. Harris, J. C. Farman, and D. W. Fahey, ibid. 89, 219801 (2002); P. K. Patra and M. S. Santhanam, ibid. 89, 219803 (2002).

[15] R. Müller, Phys. Rev. Lett. 91, 058502 (2003).

[16] X. Li et al., J. Phys. Chem. 109, 4591 (2005).

[17] R. Müller, J. Chem. Phys. 129, 027101 (2008).

[18] C.-R. Wang et al., J. Chem. Phys. 129, 027102 (2008).

[19] J. C. Farman et al., Q. J. R. Meteorol. Soc. 111, 1013 (1985).

[20] V.E. Fioletov et al., J. Geophys. Res. 107, 4647 (2002); W. Steinbrecht, H. Claude, and P. Winkler, ibid. 109, D02308 (2004).

[21] V.E. Fioletov, J. Geophys. Res. 114, D02302 (2009).

[22] J. K. Angell, J. Clim. 2, 1404 (1989).

[23] S. Solomon et al., J. Geophys. Res. 101, 6713 (1996).

[24] I. M. Jánosi and R. Müller, Phys. Rev. E 71, 056126 (2005); P. Kiss, R. Müller, and I. M. Jánosi, Nonlinear Proc. Geophys. 14, 435 (2007).

[25] H. Lee and A. K. Smith, J. Geophys. Res. 108, 4049 (2003).

[26] M. Rex et al., J. Geophys. Res. 107, 8276 (2002); N. R. P. Harris et al., ibid. 107, 8264 (2002); S. Tilmes et al., Atmos. Chem. Phys. 4, 2181 (2004).

[27] R. Müller et al., Atmos. Chem. Phys. 8, 251 (2008).

[28] G. E. Bodeker, H. Shiona, and H. Eskes, Atmos. Chem. Phys. 5, 2603 (2005).

[29] P. A. Newman and E. R. Nash, J. Atmos. Sci. 62, 614 (2005); P. Konopka et al., ibid. 62, 848 (2005).

[30] R.W. Portmann et al., J. Geophys. Res. 101, 22991 (1996).

[31] S. Tilmes et al., Atmos. Chem. Phys. 8, 1897 (2008).

[32] P. A. Newman et al., Atmos. Chem. Phys. 7, 4537 (2007).

[33] See EPAPS Document No. E-PRLTAO-103-012950 for supplementary material. For more information on EPAPS, see http://www.aip.org/pubservs/epaps.html.

[34] M. von Hobe et al., Phys. Chem. Chem. Phys. 11, 1571 (2009); H. Y. Chen et al., Science 324, 781 (2009).

[35] A. Kiendler et al., J. Phys. B 29, 6217 (1996); Q.-B. Lu and T. E. Madey, Phys. Rev. Lett. 82, 4122 (1999); C.-R. Wang et al., J. Chem. Phys. 128, 041102 (2008).

[36] K. A. Walker et al., Geophys. Res. Lett. 32, L16S04 (2005).

[37] Q.-B. Lu and L. Sanche, J. Chem. Phys. 115, 5711 (2001). 